\section{THE SMALLEST ELECTRIC ON THE MARKET}

Brush-Baby, children's dental care specialist, has launched the Brush-Baby BABYSONIC - the smallest toothbrush on the market.

For parents familiar with toddlers demanding to use an electric toothbrush, and for infants and toddlers who find a conventional toothbrush too large and unwieldy, Brush-Baby's new BABYSONIC provides a welcome solution.

The lightweight 'baby-sized' BABYSONIC is significantly smaller than regular kids' electric toothbrushes and has a soft-bristled head no bigger than the nail of a little finger.

Switch on the BABYSONIC and you'll be struck how gently the tiny brush head vibrates, fitting comfortably around tiny deciduous teeth. The easi-grip handle makes it simple for the carer to manoeuvre it over teeth and gums and a brush head which lights up helps to guide the way.

The BABYSONIC's two-minute timer helps parents teach toothbrushing. Simply divide the mouth into four sections, switch on the BABYSONIC and gently brush teeth and gums, moving on to the next section when the brush pulses (every 30 seconds). Once two minutes are complete, the brush turns off.

Brush-Baby’s BABYSONIC has an rrp of $£ 8.99$, which includes one AAA battery and one additional brush head. Unlike many kids' electric toothbrushes, replaceable brush heads are also available (pack of two, rrp $£ 3.50$ ) ensuring good value and an invaluable investment in establishing an oral care regime early.

For Brush-Baby products sales email sales@brushbaby.co.uk or call 08455202229.

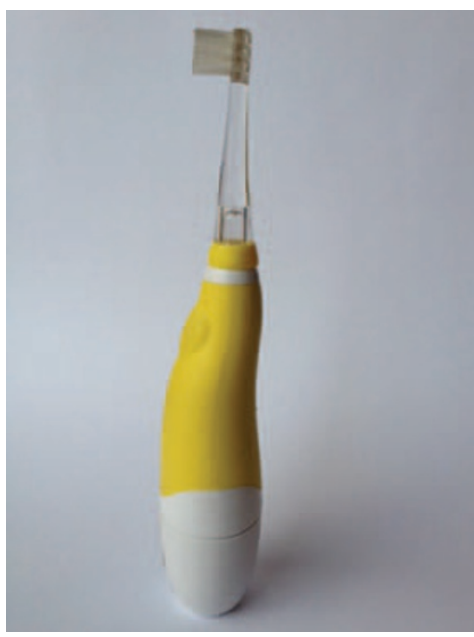

\section{MATERIALS KNOWLEDGE ROADMAP}

The Materials Knowledge Transfer Network (KTN) has recently published a roadmap of materials development for the dental industry. Entitled 'Materials in Dentistry', the roadmap was showcased at the recent 22nd European Dental Materials conference held in Birmingham. The report highlights the crucial role that polymers, metals, glass, ceramics and composites can play in the multi-disciplinary development of enhanced dental products. Rather than being prescriptive, the roadmap lists sector drivers, enablers and materials solution providers as a means of encouraging readers to make connections and so initiate novel R\&D collaborations.

A number of strong themes have emerged as the Materials KTN engaged with the dental community. For example, there is strong support for developing additive manufacture routes for ceramic/glassceramic crowns. Other topics that are frequently mentioned include longer life, self-mineralising fillings, and sensors in the mouth to monitor bacterial levels, soft tissue health etc. The roadmap (available as a hard copy and pdf from the Materials KTN) is seen as an ongoing development. The Materials KTN also welcomes further feedback and is committed to issuing subsequent versions of the roadmap to reflect any emerging intelligence.

For further information email phil.jackson@materialsktn. net. Those interested in joining the Materials KTN can do so at: https://connect.innovateuk.org/ web/materialsktn/overview.

\section{NEW SMALL \\ DIAMETER IMPLANTS}

Henry Schein Dental's miniMARK small diameter implants are ideal for stabilising lower dentures.
Each implant design features a tapered thread and sharp pinpoint tack tip to improve soft tissue penetration and insertion and aid immediate fixation. Surface texturing as standard improves the speed and effectiveness of osseointegration.

Available in three differing sizes, the $2.3 \mathrm{~mm}$

\section{AWARD-WINNING FIBRE-FREE LASER}

Syneron Dental Lasers has been selected among the winners of the international Red Dot Award, for product design in the Science and Medicine Category.

LiteTouch laser was awarded the internationally renowned Red Dot Design Award, which is the largest and most distinguished design competition award with over 4,700 entries from 54 countries within 19 different product categories against key criteria including the degree of innovation, ergonomics, durability and ecological soundness.

The Red Dot jury selected LiteTouch as the award winner due to its fibre-free laser delivery technology, which is easy for dentists to use and has enhanced hard and soft tissue treatments.

Syneron Dental Lasers is the inventor of groundbreaking LiteTouch, the most versatile dental laser for hard and soft tissue treatments. Thanks to miniaturisation of its laser technology the whole power generator is incorporated within the Laser-in-Handpiece mechanism. This solution mimics the feel of the turbine drill, yet incorporates the unique benefits of a laser: micro surgery, quicker healing, minimal invasive treatments and higher patient acceptance of dental treatments.

For more information, go to www.synerondental.com.

and $2.9 \mathrm{~mm}$ implants have Zest LOCATOR Heads that can be placed in a two-step procedure, making immediate chairside denture retention possible.

Call 08700102043. 